\title{
Using Game Engines in Mixed Reality Installations
}

\author{
Marija Nakevska, Casper Vos, Alex Juarez, Jun Hu, Geert Langereis, \\ and Matthias Rauterberg \\ Department of Industrial Design, Eindhoven University of Technology \\ m.nakevska@tue.nl, e.c.vos@student.tue.nl, \\ \{acordova, g.r.langereis, j.hu, g.w.m.rauterberg\} @ tue.nl
}

\begin{abstract}
In mixed reality installations we have to integrate a variety of technologies such as virtual reality, augmented reality, animated virtual agents and robotic agents. In this paper we describe some of our explorations with a game engine as the driving software for mixed reality installations.
\end{abstract}

Keywords: Mixed reality, virtual reality, CAVE, game engine.

\section{Introduction}

In a mixed reality installation, a variety of technologies is integrated such as virtual reality, augmented reality, and animated virtual agents and robotic agents. One of the main challenges is how to design and implement a mixed reality installation that integrates a heterogeneous array of sensors and actuators, immersive interactive environments into aware system that will engage the user, providing a meaningful experience.

In this paper we address the problems of building a CAVE environment in mixed reality installations, and share our experience in using a game engine as the driver for the CAVE, and in interfacing the game engine with sensors, actuators and input devices.

\section{Game Engines for the CAVE}

A CAVE system is usually a projection based virtual reality environment, consisting of a room in which the walls, ceiling and floor surround a viewer with projected images [1]. Building realistic virtual environments is a complex, expensive and time consuming process. Although virtual environment development toolkits are available, many just provide a subset of the tools needed to build complete virtual worlds. One alternative is the reuse of computer game technology. The current generation of computer games presents realistic virtual worlds featuring user-friendly interaction and the simulation of the real world. A number of computer game engines provide tools, documentation and source code, either with the game itself or separately available, so the end-users can create new content.

CryEngine [2] supports a number of features that are useful for creating immersive and realistic games and virtual environments, the necessary development tools are 
integrated with the engine itself, including CryEngine Sandbox world editing system. Advantages to using the CryEngine include the fact that the engine produces very high quality graphics and visuals. Disadvantages with this engine include that it has high demands on supporting hardware requirements for the high visual and audio components.

In previous work we introduced the CryEngine automatic Virtual Environment (CryVE), a CAVE system based on the game engine CryEngine2 [3]. The software architecture uses the multiplayer features of a CryEngine computer game to build the projections in the different sides of the installation. Instances of the game are started on all computers in the system, connected to each other through a network hub, with one of them acting as a server (master) while the rest are game clients (slaves). The server controls the interactive, "in-game" action (walking, jumping, flying, etc.) while the clients provide the extra "cameras" that complete the peripheral view required by the CAVE, by aligning and synchronizing themselves to the pose and motion of the master, effectively enlarging its field of view. Finally each computer renders its piece of the virtual world to the corresponding projection screen (Fig.1 shows the CryVE system architecture). A more detailed description of the design and implementation of CryVE can be found in [4].

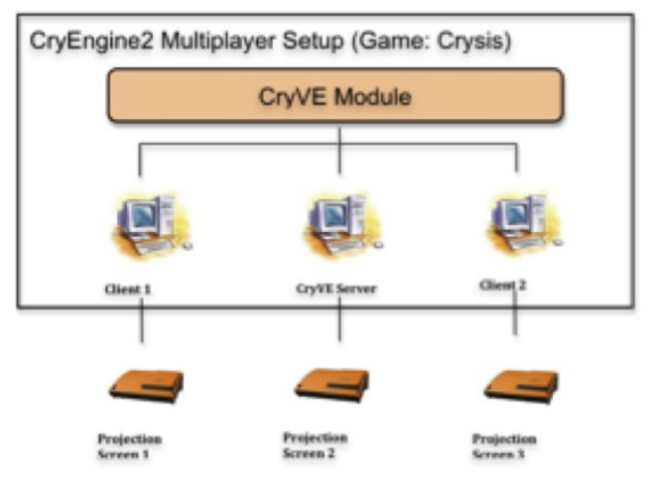

Fig. 1. CryVE system architecture (taken from [cite journal paper])

\section{Use cases}

Next we describe some of our explorations with the utilizing game engines in mixed reality installations.

\subsection{The ALICE Project and Cultural Computing}

The ALICE installation [5] uses mixed reality as a new medium for edutainment and entertainment, and consists of six consecutive stages, using a variety of technologies such as virtual reality, augmented reality, animated virtual agents and robotic agents. One of the stages in the ALICE project is based on the book chapter where Alice adjusts her size based on eating and drinking. It is associated with the concept of 
space: a CAVE system is used to be able to manipulate the relative size of the users in comparison to the environment. The virtual room is projected on a five sided CAVE, the bottle features touch and tilt sensors to detect drinking and the cookie box is equipped with a microphone that allows detecting the visitor's chewing sounds when eating the cookie. The implementation of interaction with the game engine is done with XML messaging.
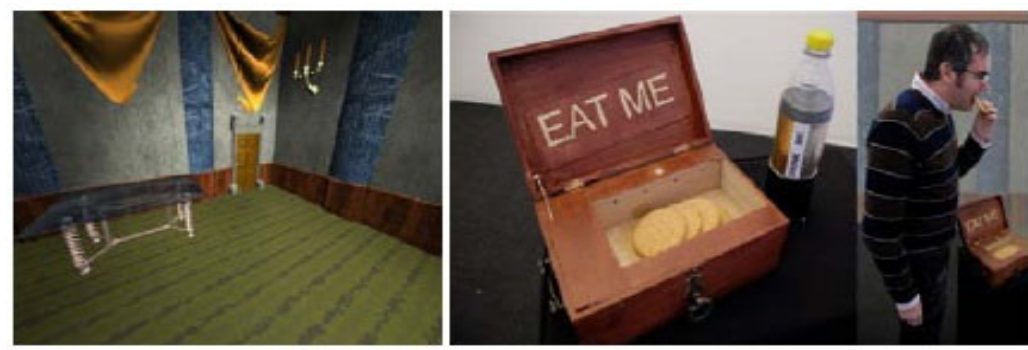

Fig. 2. In the ALICE installation [2,3], the "Eat me, Drink me" stage is implemented in a CAVE system

\subsection{A Virtual Museum Tour}

We developed a conceptual design of a Virtual Museum Exhibition that showcased historic events from the Netherlands [6]. The virtual environment resembled a medieval settlement, a virtual world that recreated sixteenth century Holland. For this, a medieval game map was modified by adding dynamic interactions that could be selected by the visitors. Visitors of the museum can use a portable controller in combination with CryVE to have an immersive experience. The handheld device is used as an interface for exploring the virtual world.
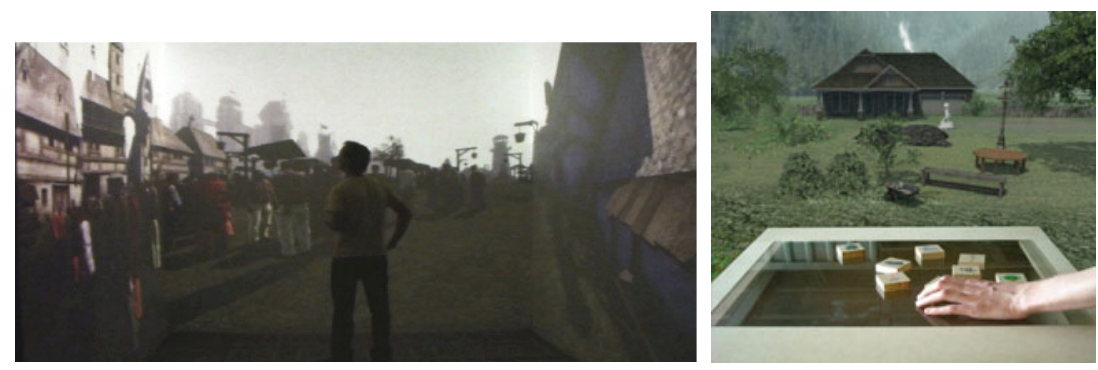

Fig. 3. A tour in the Virtual Museum (left) Impression of the Virtual Garden(right)

\subsection{Virtual Garden with Tangible Interfaces}

The Virtual Garden project is an attempt to aid a common person in designing his or her garden, without requiring the user to be proficient with computers. A CryEngine driven CAVE is used to create a convincing virtual environment. The user can 
explore and manipulate this environment through a tangible interface, which is basically a miniature representation of the virtual environment. The physical objects of the interface are fitted with pattern markers which determinate the position and rotation of the object from the video stream and are transferred to an XML file where each of the objects is a child with three attributes (xpos, ypos, rotation).

\section{Discussions and Future Work}

We have developed different implementations of mixed reality environments with the CryVE system. The presented solutions are using the same software architecture for synchronization of the projection but different ways for interaction with the virtual world have to be developed. In each of the mixed reality installations we have to integrate different sensors, actuators and input devices. Interaction with hardware is implemented by means of XML packet exchange.

With the CryVE system we have gained a platform for easy development of virtual environments, the future challenge is to develop a platform which will easily integrate different inputs from the mixed reality environment. Problems which have to be solved are synchronization of the frame buffers, integration of different input devices and flexibility in incorporation of different sensors, actuators and input/output devices.

\section{References}

[1] Cruz-Neira, C., Sandin, D., DeFanti, T., Kenyon, R., Hart, J.: The Cave: Audio Visual Experience Automatic Virtual Environment

[2] Seeley, H.: Game technology 2007: Cryengine2. In: ACM SIGGRAPH 2007 Computer Animation Festival, p. 64. ACM, New York (2007)

[3] Crytek: Cryengine2 Specifications, http: / / crytek. com/cryengine/cryengine2 / overview (web view on April 22, 2011)

[4] Juarez, A., Schonenberg, W., Bartneck, C.: Implementing a Low-Cost CAVE System Using the CryEngine2. Journal of Entertainment Computing 1(3-4), 157-164 (2010)

[5] Hu, J., Bartneck, C., Salem, B., Rauterberg, M.: ALICE's adventures in cultural computing. International Journal of Arts and Technology 1(1), 102-118 (2008)

[6] Juarez, A., Schonenberg, W., Bartneck, C., Bartneck, A.: Live history - A vision for the Nationaal Historich Museum in Arnhem, Netherlands, http://nhm.id.tue.nl/ (web view on April 22, 2011) 